\title{
Critical Dynamics of a Vortex Loop Model for the Superconducting Transition
}

\author{
Vivek Aji and Nigel Goldenfeld \\ Department of Physics, University of Illinois at Urbana-Champaign \\ 1110 West Green Street \\ Urbana, IL, 61801-3080
}

(November 1, 2018)

\begin{abstract}
We calculate analytically the dynamic critical exponent $z_{M C}$ measured in Monte Carlo simulations for a vortex loop model of the superconducting transition, and account for the simulation results. In the weak screening limit, where magnetic fluctuations are neglected, the dynamic exponent is found to be $z_{M C}=3 / 2$. In the perfect screening limit, $z_{M C}=5 / 2$. We relate $z_{M C}$ to the actual value of $z$ observable in experiments and find that $z \sim 2$, consistent with some experimental results.
\end{abstract}

PACS Numbers: 05.70.Jk, 74.40.+k, 75.40.Gb, 75.40.Mg

The discovery of the short coherence length cuprate superconductors has allowed heretofore inaccessible fluctuation effects in superconductors to be probed. Beginning with the penetration depth measurements of Kamal et al. [1], and including measurements of magnetic susceptibility [2,3], resistivity [3, 价 and specific heat [5], static and dynamic fluctuation effects have been convincingly observed and accurately quantified. These measurements are consistent with the theory of a strongly type-II superconductor, with a weak coupling of the order parameter to the electromagnetic field, described by the 3D XY model coupled to a gauge field [6].

The dynamic critical exponent, $z$, characterizes the relaxation to equilibrium of fluctuations in the critical regime of systems exhibiting a second order phase transition $[7,8]$. In particular it relates the time scale of relaxation, $\tau$, to a relevant length scale, $x: \tau \sim x^{z}$. For infinite systems $x$ is the correlation length, $\xi$. Near the critical point, the correlation length diverges and the relaxation time tends to infinity, a phenomenon known as critical slowing down. In finite size scaling studies, $x$ is identified as the system size $L$.

The dynamic critical exponent, obtained from the measurement of longitudinal dc-resistivity for YBCO is $z=1.5 \pm 0.1$ in finite but small magnetic fields [9]. Similar results were reported for the zero-field DC conductivity 10,11. Frequency dependent microwave conductivity experiments yield $z \sim 2.3-3.0[12$. On reanalysis it was found that the data were consistent with $z \sim 2$ provided one neglected the region close to $T_{c}$ [13]. Moloni et al. obtained $z=1.25 \pm 0.05$ at low magnetic fields [14], but a later, more complicated analysis by these authors gave $z=2.3 \pm 0.2$. More recently, DC conductivity measurements on single crystal BSCCO samples were interpreted to give evidence for $z \sim 2$ [15]. In summary, experiments do not yet yield a consistent picture of the critical dynamics.

If the dynamic exponent were indeed $z \sim 1.5$, then this would be surprising. Precisely this value is obtained for the superfluid transition in $\mathrm{He}^{4}$ where the combination of second sound (a propagating mode, therefore $z=1$ ) and order parameter dynamics (diffusive, therefore $z \sim 2$ ) lead to $z=3 / 2$ (model $\mathrm{E}$ dynamics) [7]. In YBCO, however, the combination of a momentum sink arising from the lattice, and the Coulomb interaction destroying the longitudinal current fluctuations should lead to pure order parameter dynamics and a prediction that $z \sim 2$ (model A dynamics). It is of course possible that some other mechanism can yield $z \sim 1.5$.

To shed light on these issues the critical dynamics was investigated numerically by performing a Monte Carlo calculation of $z$ for the 3-dimensional XY model, in the vortex representation (the so-called Villain model [16]), with and without magnetic screening [17]. The spin wave degrees were replaced by discrete vortex variables and the dynamics imposed was dissipative. The dynamic exponent estimated through a scaling analysis of the resistivity calculated within linear response will be denoted by $z_{M C}$. Surprisingly enough the exponent was found to be $z_{M C} \sim 1.5$ when the interaction was unscreened while $z_{M C} \sim 2.7$ in the presence of screening. Not only does the value $z_{M C} \sim 1.5$ agree with previous results obtained by performing a similar analysis on the London Lattice Model (LLM) 18] but also with the value of $z$ reported in some of the experiments cited above. The observations in the computer simulations are surprising because there are no collective modes in the Villain model so that the dynamics would be expected to be purely diffusive, with $z_{M C} \sim 2$. Nevertheless, and contrary to expectation, here too the system seems to support model E dynamics. Other extensive simulation studies report values of $z_{M C} \sim 1.5$ and $z_{M C} \sim 2$ depending upon the boundary conditions 19.

The purpose of this Letter is to calculate analytically the dynamic exponent for the Villain model. The equation of motion, corresponding to the Monte Carlo steps implemented in the numerical computation, is derived and analyzed near equilibrium. A scaling analysis is used to extract $z_{M C}$. We are able to explain the simulation results in both strong and weak screening limits. We show also that the simulation results cannot be interpreted as providing evidence in support of the $z \sim 1.5$ result found 
in some experiments, because they do not measure the true dynamic critical exponent: $z_{M C} \neq z$. We show how to relate $z_{M C}$ and $z$, and find that the result $z_{M C} \sim 1.5$ is in fact an artifact of taking the thermodynamic limit and the range of vortex interactions to infinity limit in the wrong order. The correct physical prediction from the simulation is $z \sim 2$ for any finite range of interaction, consistent with some observations.

The Villain model:- Consider the XY model with a fluctuating vector potential $\vec{a}$ represented as lattice gauge theory link variables $a_{i j} \equiv a_{i}-a_{j}$ :

$$
H=-J \sum_{\langle i, j\rangle} \cos \left(\phi_{i}-\phi_{j}-\lambda_{0}^{-1} a_{i j}\right)+\frac{1}{2} \sum_{\square}[\vec{\nabla} \times \vec{a}]^{2}
$$

where $J$ is the coupling constant, $\lambda_{0}$ is the screening length, $\phi_{i}$ is the phase of the condensate on site $i$ of a simple cubic lattice of size $N=L^{3}$ with periodic boundary conditions. The first sum is taken over nearest neighbors, while the second is over plaquettes of the lattice. The lattice spacing has been set to unity. The fluctuating gauge potential $a_{i j}$ satisfies the constraint that at each site $i$, the discrete divergence vanishes: $[\vec{\nabla} \cdot \vec{a}]_{i}=0$. The phase degrees of freedom can be replaced by vortices by introducing the periodic Villain function to replace the cosines. Standard manipulations [20] lead to the dual Hamiltonian:

$$
H_{V}=\frac{1}{2} \sum_{i, j} \overrightarrow{n_{i}} \cdot \overrightarrow{n_{j}} G_{i j}\left[\lambda_{0}\right]
$$

where the $\overrightarrow{n_{i}}$ 's are vortex variables that reside on the links of the dual lattice and $G_{i j}$ is the screened lattice Green's function,

$$
G_{i j}\left[\lambda_{0}\right]=J \frac{(2 \pi)^{2}}{L^{3}} \sum_{\vec{k}} \frac{\exp \left[i \vec{k} \cdot\left(\overrightarrow{r_{i}}-\overrightarrow{r_{j}}\right)\right]}{2 \sum_{m}^{3}\left[1-\cos \left(k_{m}\right)\right]+\lambda_{0}^{-2}}
$$

The two limits that are considered in the simulations are the long range case, $\lambda_{0} \rightarrow \infty$, and the short range case, $\lambda_{0} \rightarrow 0$. Actually the simulations were performed by setting $\lambda_{0}=0$ and $\lambda_{0}=\infty$ in (3). The distinction between the limit and the actual simulations will turn out to be significant. In both cases the local constraint of no monopoles, $[\vec{\nabla} \cdot \vec{n}]_{i}=0$, is imposed. Each Monte Carlo move consists of trying to create a closed vortex loop around a plaquette. The trial state is accepted or rejected according to the heat bath algorithm with probability $1 /[1+\exp (\beta \Delta E)]$ where $\Delta E$ is the change in energy and $\beta=1 / k_{B} T$, with $k_{B}$ being Boltzmann's constant. Each time a vortex loop is formed it generates a voltage pulse, $\Delta Q= \pm 1$, perpendicular to its plane, the sign depending on the orientation. This voltage fluctuation gives rise to an electrical resistance, $R$, which can be analyzed within linear response theory. A point that will be important to note here is that $R$ depends on the average change in the total number of loops pointing in a given direction at each time step. The unit of time is normalized so that, on average, an attempt has been made to create or destroy one loop per plaquette.

Dipole gas description:- It is known that near $T_{c}$ the static properties are dominated by the proliferation of vortex loops of unit strength, i.e., it is energetically unfavorable to create vortex loops of greater strength at each plaquette. The interaction between these vortex loops is spherically symmetric and so is the state in thermal equilibrium. As it stands, the computations above have been performed on what is known as the low temperature Villain model and the critical point is obtained by looking at the intersection of the low and high temperature Villain models (for details see ref. [20]). The physics described here is that of an interacting gas of dipoles, $\vec{d}$. In the long range case they interact via the standard Coulomb term which falls off as $1 / r^{3}$; note that these dipoles interact antiferromagnetically, and are not current loops, which interact via the standard ferromagnetic interaction.

For our analysis we shall consider a cubic lattice, $L^{3}$, on whose vertices reside the loop variables, $\overrightarrow{l_{i}}$. In terms of the vortex variables $\overrightarrow{n_{i}}=\vec{\nabla} \times \overrightarrow{l_{i}}$, as can be seen by writing out the components. The three components are each either \pm 1 or 0 , corresponding to a clockwise, anticlockwise or absence of a vortex loop along the three principle directions, $x, y$ or $z$. The corresponding probabilities on site $i$ at time step $s$ are given by $P_{i s}^{\alpha}[1], P_{i s}^{\alpha}[-1]$ and $P_{i s}^{\alpha}[0]$, where $\alpha$ is a coordinate label. The quantity computed in the simulations is the total number of loops, $N_{s}^{\alpha}$ pointing along a given direction $\alpha$ at time step $s$.

$$
N_{s+1}^{\alpha}=\sum_{i}\left(P_{i s+1}^{\alpha}[1]-P_{i s+1}^{\alpha}[-1]\right)
$$

To study the behavior of $N_{s}^{\alpha}$, we follow the standard procedure of writing out the master equation for the time development of the probabilities and evaluating (4) [21]. As previously indicated, the equilibrium state is spherically symmetric. That is, on average, $\Delta E_{i s}^{\alpha}$, the change in energy on adding a unit loop on site $i$ at time $s$, is zero. This implies that transition probabilities for creating and annihilating a vortex loop are equal. The heat bath algorithm ensures that the conditions of detailed balance are satisfied. Furthermore, at $T_{c}$, the restriction to unit loops per plaquette results in $P_{i s}^{\alpha}[0]=P_{i s}^{\alpha}[1]=P_{i s}^{\alpha}[-1]$ in equilibrium. Since we are interested in small deviations from equilibrium, we impose a uniform perturbation, $\delta l^{\alpha}$ per site and see how it relaxes back to equilibrium. This implies $\delta N^{\alpha} \sim L^{3} \delta l^{\alpha}$. To leading order the equation of motion reads

$$
\frac{d \delta N^{\alpha}}{d t}=-\frac{2}{3} \beta \sum_{i} a_{i}^{\alpha}\left(\frac{\partial \Delta E_{i}^{\alpha}}{\partial l^{\alpha}}\right)_{0} \delta l^{\alpha}
$$

where the subscript 0 denotes equilibrium, and $a_{i}^{\alpha}$ is the transition probability in equilibrium for creating the dipole loops.

Scaling analysis:- Equation (5) is the basis for the scaling analysis that follows. The only relevant length scales 
are the system size, $L$, and the correlation length, $\xi \cdot a_{i}^{\alpha}$ is an equilibrium microscopic transition probability which remains finite at the critical point while $\beta \sum_{i} \Delta E_{i}^{\alpha}$ is dimensionless and scales as $(L / \xi)^{3}$ away from $T_{c}$ for finite systems. This follows because by definition, thermodynamic additivity occurs on a scale beyond the correlation length. While the free energy is extensive for all temperatures, at $T_{c}, \xi \sim L$ and $\beta \sum_{i} \Delta E_{i}^{\alpha}$ is independent of $L$. Thus the characteristic time scale of relaxation of the perturbation, $\tau$, scales as

$$
\tau \sim \frac{\xi^{3}[l]}{a_{i}^{\alpha}}
$$

where $[l]$ is the scaling dimension of the field $l$.

For the long range case the binding energy is given by,

$$
\beta H=-\beta \sum_{i, j} \frac{\vec{d}_{i} \cdot \vec{d}_{j}-3\left(\vec{d}_{i} \cdot \hat{r}_{i j}\right)\left(\vec{d}_{j} \cdot \hat{r}_{i j}\right)}{r^{3}}
$$

where $\vec{d}_{i}=\mu \vec{l}_{i}, \mu$ is the dipole strength of a unit loop around a plaquette, $r=\left|\vec{r}_{i j}\right|$, where $\vec{r}_{i j}=\overrightarrow{r_{i}}-\overrightarrow{r_{j}}$ and $\hat{r}_{i j}$ is the unit vector along $\vec{r}_{i j}$. If $\vec{l}_{i}$ were dimensionless, then the energy of the system would not be extensive. To evaluate the dimension of $\vec{l}$ note that $L^{6}[l]^{2} / \xi^{3} \sim(L / \xi)^{3}$ as required by the extensivity of the free energy. Thus $[l] \sim L^{-3 / 2}$ and $\tau \sim \xi^{3} L^{-3 / 2}$. The dynamic exponent at $T_{c}$, where $\xi=L$, in this case is $z_{M C}=3 / 2$, which is consistent with the computer simulation results.

For the short range case the binding energy is given by

$$
\beta H=\beta \sum_{i} \overrightarrow{n_{i}} \cdot \overrightarrow{n_{i}}=\beta \sum_{i}\left(\vec{\nabla} \times \vec{d}_{i}\right) \cdot\left(\vec{\nabla} \times \vec{d}_{i}\right)
$$

Requiring extensivity, i.e. $[l]^{2} \xi^{-2} L^{3} \sim(L / \xi)^{3}$, yields $[l] \sim \xi^{-1 / 2}$. From (6) we get $\tau \sim \xi^{5 / 2}$ which at $T_{c}$ scales as $L^{5 / 2}$. The dynamic exponent is $z_{M C}=5 / 2$, which is consistent with the computer simulation results 17].

Critical dynamics of the dipole gas model:- We will now derive the governing stochastic partial differential equation that describes the long wavelength critical dynamics of the superconductor. Our strategy will be to first derive the continuum limit of the Hamiltonian (2), and then impose relaxational dynamics. We will find that the results for $z$ are not the same as our results for $z_{M C}$. This is because the Monte Carlo time step does not correspond to the physical time step. This is explained below. Let us first look at the continuum limit of the short range case. Reintroducing the coupling constants and the lattice spacing, $a$, we write the Hamiltonian $H_{V}$ for the vortex variables as

$$
H_{V}=J\left(2 \pi \frac{\lambda_{0}}{a}\right)^{2} \sum_{i}\left(\vec{\nabla} \times \overrightarrow{l_{i}}\right) \cdot\left(\vec{\nabla} \times \overrightarrow{l_{i}}\right)
$$

Converting the sum to an integral,

$$
H_{V}=-\left(J / a^{3}\right)\left(2 \pi \frac{\lambda_{0}}{a}\right)^{2} \int d \vec{r}(\vec{\nabla} \times \vec{l}(\vec{r})) \cdot(\vec{\nabla} \times \vec{l}(\vec{r}))
$$

In the limit $a \rightarrow 0, J a^{-3} \rightarrow \widetilde{J}$ and $\lambda_{0} / a \rightarrow \widetilde{\lambda_{0}}$. Relaxational dynamics is governed by the time-dependent Ginzburg-Landau equation (TDGL), which in this case is

$$
\frac{\partial \vec{l}}{\partial t}=\Gamma \widetilde{J}\left(2 \pi \widetilde{\lambda_{0}}\right)^{2}\left(\nabla^{2} \vec{l}-\vec{\nabla}(\vec{\nabla} \cdot \vec{l})\right)+\vec{\eta}
$$

where $\eta$ is a white noise, satisfying the fluctuation dissipation theorem with $\left\langle\eta^{\alpha}(\vec{r})\right\rangle=0$ and $\left\langle\eta^{\alpha}\left(\vec{r}^{\prime}\right) \eta^{\beta}(\vec{r})\right\rangle=$ $2 \Gamma k_{B} T \delta_{\alpha \beta} \delta\left(\vec{r}^{\prime}-\vec{r}\right)$. The TDGL equation is similar to the diffusion equation and is expected to yield a dynamic exponent of $z=2$, in mean field theory, with small corrections due to fluctuations. The linearity of the TDGL in this case reflects the fact that only unit vortices are considered in the analysis.

In the long range case, taking the continuum limit, we obtain

$$
\begin{aligned}
& H_{V}=\widetilde{J}(2 \pi)^{2} \int d \vec{r}^{\prime} d \vec{r} \frac{\left(\vec{\nabla} \times \vec{l}\left(\vec{r}^{\prime}\right)\right) \cdot(\vec{\nabla} \times \vec{l}(\vec{r}))}{\left|\vec{r}^{\prime}-\vec{r}\right|} \\
& \times \exp \left[-\left|\vec{r}-\vec{r}^{\prime}\right| / \lambda_{0}\right]
\end{aligned}
$$

where the infinite self energy has been subtracted, and the screening length $\lambda_{0}$ is taken to be finite. To relate this to the dipole-dipole interaction used in our analytic model of the simulations, consider a cubic lattice, as before, on whose vertices sit variables $\vec{d}_{i}$, and take $\lambda_{0}=\infty$. Replacing $2 \pi \sqrt{\widetilde{J}} l(\overrightarrow{\vec{r}})=\sum_{i} \vec{d}_{i} \delta\left(\vec{r}-\overrightarrow{r_{i}}\right)$ one can perform the integrals over $\vec{r}$ and $\overrightarrow{r^{\prime}}$, to recover the expression in (7). The actual TDGL equation for the long range case reads

$$
\begin{aligned}
\frac{\partial \vec{l}(\vec{r})}{\partial t}=\Gamma \widetilde{J}(2 \pi)^{2} \int d \vec{r}^{\prime} & \frac{\nabla^{2} \vec{l}\left(\vec{r}^{\prime}\right)-\vec{\nabla}\left(\vec{\nabla} \cdot \vec{l}\left(\vec{r}^{\prime}\right)\right)}{\left|\vec{r}^{\prime}-\vec{r}\right|} \\
& \times \exp \left[-\left(\left|\vec{r}-\vec{r}^{\prime}\right| / \lambda_{0}\right)\right]+\vec{\eta}
\end{aligned}
$$

Let us first take the case $L \rightarrow \infty$ with $\lambda_{0}$ finite but large. The dynamic exponent in this case is 2 , because the kernel effectively renormalises the time scale in a way that is independent of system size. If we took the two limits $L \rightarrow \infty$ and $\lambda_{0} \rightarrow \infty$ in the opposite order, as was done in the computer simulations, the exponential factor would not be present, and the dynamics would be independent of $L$. Hence the dynamic exponent would then be $z=0$.

Nature of the long range case:- The rather curious result of $z=0$ is obtained for the situation where the screening length is sent to infinity before taking the thermodynamic limit. Whether the interaction is considered short or long range depends on with what it is compared. Physically the short range case describes the situation where $\lambda_{0}$ is much smaller than the inter-vortex spacing 
$\lambda_{v}$. This is indeed captured in the simulations by setting $\lambda_{0}=0$. Physically the long range case describes the situation where $L \gg \lambda_{0} \gg \lambda_{v}$. This is not captured by setting $\lambda_{0}=\infty$ with $L$ finite.

Reconciliation with the lattice model simulations:- The critical dynamics of the lattice simulations and the continuum analysis above do not apparently agree. We now will show that this is because the time step in the simulation does not correspond to the physical time step. The reason is that from the definition of the loop variable $\overrightarrow{l_{i}}$, the net electric field at time $t$ is $\mathrm{E}^{\alpha}(t)=\sum_{i} l_{i}^{\alpha} d P\left[l_{i}^{\alpha}\right] / d t$. In the simulations, and in (4), this has been replaced by $\mathrm{E}^{\alpha}\left(t_{M C}\right)=\sum_{i} d P\left[l_{i}^{\alpha}\right] / d t_{M C}$, where $t_{M C}$ denotes the Monte Carlo time and $l_{i}^{\alpha}= \pm 1,0$ only. However in the long range case and in the short range case at $T_{c}$, where $\xi=L,[l]$ depends on $L$. Hence the physical time is related to the Monte Carlo time by $t=t_{M C}[l]$ so that the relaxation time is actually

$$
\tau \sim L^{3}[l]^{2} / a_{i}^{\alpha} .
$$

The dynamic exponents for the lattice model are then $z=2$ for the short range case and $z=0$ for the long range case, in agreement with the analytic calculation based on the continuum limit equations of motion. We see that the simulation result $z_{M C}=3 / 2$ or equivalently its corrected form $z=0$ arise from taking the thermodynamic limit and the long-range of interaction limits in the incorrect order. With this correction to the results of the simulation, the results no longer are consistent with those experiments reporting $z \sim 1.5$.

Experimental ramifications:- In experiments performed on bulk superconductors one would expect the short range limit of the model above to apply, provided that the interaction range is shorter than the system size. In such systems, as long as diffusive dynamics for the vortex degrees of freedom is applicable, a dynamic exponent of 2 is predicted by the model above.

What then could be the origin of the behaviour $z \sim 1.5$, if confirmed, in some experiments? There are at least two possible avenues for further investigation into the true nature of the critical dynamics in these systems. The first is to seek experimental evidence for the existence of hydrodynamic modes which might account for the observed model E dynamics in transport properties. The $41 \mathrm{meV}$ peak observed in neutron scattering data is one possible candidate 22] although it does not seem to occur near the origin, while certain interpretations of the peak-diphump structure seen in ARPES are also suggestive of the existence of a collective mode in the system [23]. A second possibility is to study the crossover from model $\mathrm{E}$ to model A dynamics as the effective coupling of the condensate with the electromagnetic field tends towards zero (equivalently one can study the crossover by sending the plasmon gap to zero).

In conclusion, we have explained the simulation results for the critical dynamics of the superconducting transition in zero field, and shown that in fact they are consistent with expectations based on the TDGL. An extension of this analysis to two dimensions will be presented elsewhere.

\section{ACKNOWLEDGMENTS}

We thank Jack Lidmar, Mats Wallin, Peter Young and Steve Teitel for helpful comments on an earlier version of this manuscript. We acknowledge support from the National Science Foundation through grant NSF-DMR99-70690.

[1] S. Kamal, D. Bonn, N. Goldenfeld, P. Hirschfeld, R. Liang and W. N. Hardy, Phys. Rev. Lett. 73, 1845 (1994).

[2] R. Liang, D. A. Bonn and W. N. Hardy, Phys Rev. Lett. 76, 835 (1996).

[3] M.B. Salamon, J. Shi, N. Overend and M. Howson, Phys. Rev. B 47, 5520 (1993).

[4] M.A. Howson, N. Overend, I.D. Lawrie and M.B. Salamon, Phys. Rev. B 51, 11984 (1995).

[5] N. Overend, M.A. Howson and I.D. Lawrie, Phys. Rev. Lett. 72, 3238(1994).

[6] D.S. Fisher, M.P.A. Fisher and D.A. Huse, Phys. Rev. B 43, 130 (1990).

[7] P.C. Hohenberg and B.I. Halperin, Rev. Mod. Phys. 49, 435 (1977).

[8] See (e.g.) Nigel Goldenfeld, Lectures on Phase Transitions and the Renormalization Group (Addison-Wesley, Reading MA, 1992).

[9] J.T. Kim, N. Goldenfeld, J. Giapintzakis and D.M. Ginsberg, Phys. Rev B 56, 118 (1997).

[10] W. Holm, Yu Eltsev and Ö. Rapp, Phys. Rev B 5111 992 (1995).

[11] A. Pomar et al., Physica C 218257 (1993).

[12] J.C. Booth et al,. Phys. Rev. Lett. 77, 4438 (1996).

[13] R.A. Wickham and A.T. Dorsey, Phys. Rev. B 61, 6945 (2000).

[14] K. Moloni et al., Phys. Rev. Lett. 78, 3173 (1997); ibid. Phys. Rev. B 56, 14784 (1997).

[15] S.H. Han, Yu. Eltsev and Ö. Rapp, J. Low Temp. Phys. 117, 1259 (1999).

[16] J. Villain, J. Phys. (Paris) 36, 581 (1977).

[17] J. Lidmar et al., Phys. Rev. B 58, 2827 (1998).

[18] H. Weber and H.J. Jensen, Phys. Rev. Lett. 78, 2620 (1997).

[19] L.M. Jensen, B.J. Kim and P. Minnhagen, Phys. Rev. B 61, 15412 (2000); see also, P. Minnhagen, B.J. Kim and H. Weber, cond-mat/0105323.

[20] H. Kleinert, Gauge theories in condensed matter, Vol. 1 (World Scientific, 1989).

[21] See (e.g.) A. Isihara, Statistical Physics (Academic, 1971).

[22] P. Bourges et al., Science 288, 1234 (2000).

[23] M.R. Norman and H. Ding, Phys. Rev. B 57, R11089 (1998). 